\title{
Caracterización y modelación hidrogeoquímica de lixiviados mineros de San Luis Potosí, S.L.P. México
}

\author{
Juan Carlos Burillo, Antonio Cardona, Javier Castro-Larragoitia, Isidro Montes
}

Juan Carlos Burillo

jcburillo@yahoo.com.mx

Antonio Gardona

Javier Castro-Larragoitia

Isidro Montes

Área de Ciencias de la Tierra de la Facultad de Ingeniería, Universidad Autónoma de San Luis Potosí. Av. Dr. Manuel Nava \# 8 Edificio G. Zona Universitaria, San Luis Potosí, S.L.P. 78290 .

BOL. SOC. GEOL. MEX. 2017

VOL. 69 NO. 3

P. $637-654$

Manuscrito recibido: Febrero 15, 2017 Manuscrito corregido: Junio 10, 2017 Manuscrito aceptado: Junio 23, 2017

\section{RESUMEN}

Se empleó la modelación hidrogeoquímica para entender las diferencias entre los lixiviados mineros y los extractos acuosos de sedimentos de arroyo y de residuos sulfurosos de un distrito minero histórico de San Luis Potosí. Tanto en los lixiviados colectados en campo como en los extractos acuosos obtenidos en laboratorio predominaron las especies de $\mathrm{Al}, \mathrm{Ca}, \mathrm{Fe}, \mathrm{SO}_{4}$ y $\mathrm{Zn}$. La concentración de estas especies acuosas en los extractos de residuos fue mayor que en los sedimentos. Los residuos se presentan en dos tipos de condiciones: libres sin ningún tipo de protección y semiconfinados. La concentración en los extractos de residuos expuestos a la intemperie fue mayor que en los semiconfinados. La DRX muestra la presencia de jarosita, lo que se corresponde con los resultados de la modelación hidrogeoquímica en los extractos acuosos y lixiviados. La reacción de la calcita con los lixiviados produjo la disolución-precipitación de los minerales: gibbsita $\mathrm{Al}(\mathrm{OH})_{3}$, yeso $\left(\mathrm{CaSO}_{4} \cdot 2 \mathrm{H}_{2} \mathrm{O}\right)$, galena $\left(\mathrm{PbSO}_{4}\right)$, alunita $\mathrm{KAl}_{3}(\mathrm{OH})_{6}\left(\mathrm{SO}_{4}\right)_{2}$ y melanterita $\mathrm{FeSO}_{4}: 7 \mathrm{H}_{2} \mathrm{O}$ que es función de la cantidad de calcita presente y el pH de la solución. En general, el Fe existe como $\mathrm{Fe}^{+2}$ y el $\mathrm{S}$ como $\mathrm{SO}_{4}^{-2}$. Se modeló la mezcla entre el lixiviado minero $(\mathrm{pH}<3$ y agua de lluvia lo que produjo una solución con $\mathrm{pH}$ cercano al neutro. La evaporación de lixiviados forma sulfatos metálicos, principalmente yeso y melanterita. La evolución de agua del modelo fue de bicarbonatada cálcica hacia sulfatada cálcica, probablemente debido al efecto de ión común. Los extractos acuosos más contaminados provienen de los residuos mineros, que se corresponden espacialmente con los lixiviados, reflejando la capacidad limitada de los sedimentos de arroyo para retener metales. Este trabajo muestra la importancia de complementar la caracterización de residuos, en base a las normas ambientales vigentes en México, con el fin de proponer planes de remediación mejor fundamentados.

Palabras clave: lixiviados mineros, San Luis Potosí, caracterización de residuos mineros, modelación hidrogeoquímica.

\section{ABSTRACT}

A Hydrogeochemical modelling approach was used to understand differences between mine leachates and aqueous extracts of stream sediments and sulfide mine residues from the historical mine district of San Luis Potosí. In Both solutions, leachates and aqueous extracts, $\mathrm{Al}, \mathrm{Ca}, \mathrm{Fe}, \mathrm{SO}_{4}$ and $Z n$ species predominate. Concentration of these species was higher in mine residue extracts than stream sediment extracts. Residues present two types of conditions: free without protection and semi-confined. The concentration of aqueous species from extracts of mine residues exposed to the weather was higher than semi-confined ones. $X R D$ mineralogical characterization indicates the presence of jarosites in aqueous extracts and leachates. Configuration of reaction path reaction as a titration model shows dissolution-precipitation of minerals: gibbsite $\mathrm{Al}(\mathrm{OH})$, gypsum $\left(\mathrm{CaSO}_{4} \cdot \mathrm{H}_{2} \mathrm{O}\right)$, galena $\left(\mathrm{PbSO}_{4}\right)$, alunite $\mathrm{KAl}_{3}$ $(\mathrm{OH})_{6}\left(\mathrm{SO}_{4}\right)_{2}$ and melanterite $\mathrm{FeSO}_{4}: 7 \mathrm{H}_{2} \mathrm{O}$ occurring, as a function of the quantity of calcite reacted and $p H$. In general, $\mathrm{Fe}$ exists as $\mathrm{Fe}^{2+}$ and $\mathrm{S}$ as $\mathrm{SO}_{4}^{2-}$ The use of a mix model, mine leachate $(p H<3)$ vs rain water solution, originated a near neutral $p H$ solution. Evaporation of leachates produced metallic sulfates, gypsum and melanterite, which were founded in mine residues $X R D$ analysis too. Water evolves from $\mathrm{HCO}^{3-}$ - Ca to $\mathrm{SO}_{4}^{2-}$-Ca type, probably due to common ion effect. Moreover, the most contaminated water extracts come from mine residues, which correspond to leachates spatially, reflecting the limited capacity of stream sediments to retain metals. This paper shows the importance of complement mine residue characterization, based on current environmental standards in Mexico, in order to propose best basis remediation plans.

Keywords: mine leachates, San Luis Potosi, mine residue characterization, hydrogeochemical modeling. 


\section{Introducción}

Los residuos de las operaciones minero-metalúrgicas, son uno de los más importantes generados por la actividad humana, ya que por sus características químicas y mineralógicas propias (presencia de cantidades importantes de metales y metaloides y compuestos asociados) y las del ambiente donde se localizan (geohidrológico, climático, biológico, químico), tienen el potencial de dispersar sus componentes al medio ambiente, impactando el agua, los sedimentos, suelos, así como biota. Los procesos que originan o modifican los residuos mineros representan un fenómeno complejo que primero debe comprenderse y después atenderse, analizando diversas alternativas de remediación (Razo et al., 2007; Armienta y Segovia, 2008; Nordstrom, 2011; Lottermoser, 2011; Simate y Ndlovu, 2014; Nordstrom 2015; Gutiérrez et al., 2016).

El diseño de un programa de caracterización de un sitio minero-metalúrgico contempla la toma de muestras de sedimentos, lixiviados, residuos, jales, agua superficial y/o subterránea, incluso muestras biológicas, y su análisis en el laboratorio (Castro-Larragoitia et al., 1997; Santos-Jallath et al., 2013; Carrillo-Chávez et al., 2014; Luís et al., 2015; Root et al., 2015). Los estudios de laboratorio y campo sobre residuos minero-metalúrgicos son diversos e incluyen aspectos de caracterización mineralógica: (Moreno et al., 2012; Salas, 2014; Jamieson et al., 2015), geoquímica (Lindsay et al., 2015; Klunk et al., 2015), física e hidrológica (Sánchez-España y Diez-Ercilla, 2008; Šráček et al., 2013; Schaider et al., 2014; Amos et al., 2015), así como la caracterización de sus efluentes, que en el caso de residuos conteniendo sulfuros pueden llegar a ser los denominados Drenaje Ácido de Roca (DAR) por sus siglas en inglés (Nordstrom, 2011). Con el fin de comprender el comportamiento ambiental de residuos históricos y recientes que generan DAR, se han desarrollado diferentes estrategias. Entre los estudios hidrogeoquímicos enfocados a caracterizar la atenuación natural del DAR, están el realizado por Berger et al. (2000) y que revelan el efecto de la dilución con agua de río o la capacidad de atenuación de las rocas carbonatadas; Quispe et al. (2013) explican la disolución y precipitación de fases minerales causadas por eventos estacionales de lluvia-estiaje y Corrales-Pérez y Romero (2013) estudiaron el proceso natural de atenuación de un drenaje ácido de mina (DAM), en el que la disolución de calizas favorece la precipitación de sulfatos como yeso y además de fases secundarias de hierro (jarosita). Cuando los lixiviados se intentan neutralizar es posible emplear diversos materiales adsorbentes: compósitos de magnesita-bentonita (Masindi et al., 2015), óxidos de hierro y aluminio (Otero-Fariña et al., 2015), cenizas volcánicas (Madzivire et al., 2014) etc.

Una de las preocupaciones principales asociadas a los depósitos de residuos minero- metalúrgicos no controlados, es la magnitud del impacto de sus efluentes en la calidad del agua superficial y subterránea. Una vez caracterizados tanto los residuos como el entorno geológico, es posible aplicar una metodología que considera la modelación hidrogeoquímica de las reacciones químicas entre el lixiviado y los materiales geológicos con los que está en contacto, lo que permite comprender su evolución y en todo caso atenuación. Para describir un modelo de esta naturaleza, se debe analizar el problema a resolver, las bases de datos y códigos de computadora geoquímicos disponibles y los procesos de interacción roca-agua identificados a partir de la evidencia experimental disponible (Cardona et al., 2008; Nordstrom y Campbell, 2014). Otro enfoque incluye la utilización de mediciones de campo (Eh y pH) y análisis de laboratorio (As, Zn, $\mathrm{Ca}, \mathrm{Fe}, \mathrm{S}, \mathrm{F}, \mathrm{C}, \mathrm{Al}, \mathrm{Mn}$ ) de muestras de DAR, para elaborar diagramas de estabilidad de minerales y especies acuosas predominantes en sitios contaminados (Kinniburgh y Cooper, 2004). En otro caso de estudio, presentado por Sánchez-España y Diez-Ercilla (2008) se realizó la modelación hidrogeoquímica de una salmuera hipersalina ácida que drenaba a un depósito de pirita y formaba depósitos acuosos muy concentrados de sulfuros metálicos, con lo que determinaron el estado de equilibrio de la melanterita $\left(\mathrm{Fe}_{2} \mathrm{SO}_{4} \cdot 7 \mathrm{H}_{2} \mathrm{O}\right)$ con la 
solución.

Otro tipo de enfoque considera la realización de métodos experimentales. La prueba de extracción de constituyentes tóxicos (PECT), metales y metaloides (MM), descrita en la NOM-141-SEMARNAT-2003 (Semarnat, 2004), permite identificar concentraciones en lixiviados sintéticos generados a partir de residuos mineros. Las concentraciones son comparadas con los límites máximos establecidos en la Norma Oficial Mexicana NOM-052-SEMARNAT-2005 (Semarnat, 2006), lo que permite evaluar su peligrosidad. Corrales-Pérez (2014), utilizó este enfoque para la evaluación de movilidad de $\mathrm{As}, \mathrm{Cd}, \mathrm{Pb}$ (entre otros) a partir de depósitos de jales y una obra minera inactiva en el municipio de Taxco de Alarcón, en el estado de Guerrero, México.

El objetivo del presente estudio es contribuir a la comprensión del comportamiento ambiental de unos residuos mineros históricos con estudios de evolución hidrogeoquímica, comparando químicamente los compuestos lixiviados por la prueba de laboratorio (extracto acuoso) con los que se generan en campo (condiciones naturales). Adicionalmente, la evaluación contempla realizar modelación hidrogeoquímica para el diseño de la atenuación de los lixiviados empleando: i) calcita, ii) la dilución con agua de lluvia y iii) precipitación de fases secundarias por concentración generada por procesos de evaporación.

\section{Descripción del sitio}

\subsection{LOGALIZAGIÓN}

Los residuos mineros históricos denominados Terreros Victoria (TV), se ubican en la microcuenca Cerro San Pedro, al sureste de la capital del municipio de Cerro de San Pedro, a $20 \mathrm{~km}$ al noreste de la ciudad de San Luis Potosí (Figura 1). La microcuenca forma parte de la Región Hidrológica 37 El Salado, Guenca RH37G Presa San José-Los Pilares y Otras, Subcuenca RH37Gb Presa San
José, clasificada como de tipo endorreico (Razo et al., 2007; Vázquez, 2012).

\subsection{GARACTERÍSTICAS AMBIENTALES}

La microcuenca se encuentra en el límite de las Provincias Fisiográficas Mesa Central y Sierra Madre Oriental por lo que predomina clima seco, árido y templado, con lluvias en verano, extremoso con oscilación anual de las temperaturas medias mensuales entre 7 y $14{ }^{\circ} \mathrm{C}$, con escasas lluvias en invierno. De acuerdo con los registros meteorológicos analizados (1921-2013), la temporada seca se presenta de noviembre a abril (precipitación total promedio del orden de $75 \mathrm{~mm}$ ), mientras que la temporada de lluvias es de mayo a octubre (precipitación total promedio del orden de $325 \mathrm{~mm}$ ). Las temperaturas medias son una máxima de 27 ${ }^{\circ} \mathrm{C}$ y una mínima de $12{ }^{\circ} \mathrm{C}$. La única población ubicada en la microcuenca Cerro San Pedro es la cabecera municipal, otras comunidades principalmente rurales en los alrededores incluyen a Cuesta de Campa, Monte Caldera, Portezuelo, entre otras. Forma parte de una serie de sierras bajas con orientación general NW-SE, constituidas por una serie de plegamientos que afectan rocas sedimentarias del Cretácico, coronados en ocasiones por rocas volcánicas. Se identifican escurrimientos de tipo efímero e intermitente de carácter torrencial, la microcuenca tiene una pendiente media de $26 \%$, y una integral hipsométrica de $43 \%$, lo que indica que está en estado de madurez en cuanto a la erosión se refiere. Tiene drenaje de tipo dendrítico a subdendrítico, con orden de corrientes de 4 , densidad de drenaje de $2.51 \mathrm{~km} / \mathrm{km}^{2}$ y tiempo de concentración de 1.6 horas, por lo que es una microcuenca disectada y bien drenada, además de que su respuesta a los escurrimientos es muy rápida (Rodríguez-León, 2014).

Las unidades geológicas existentes en la microcuenca incluyen rocas sedimentarias del Mesozoico: i) Formación La Peña, ii) Formación Cuesta del Cura, que constituyen el complejo basal o basamento (Aranda, 1989) y una cubierta Cenozoica 


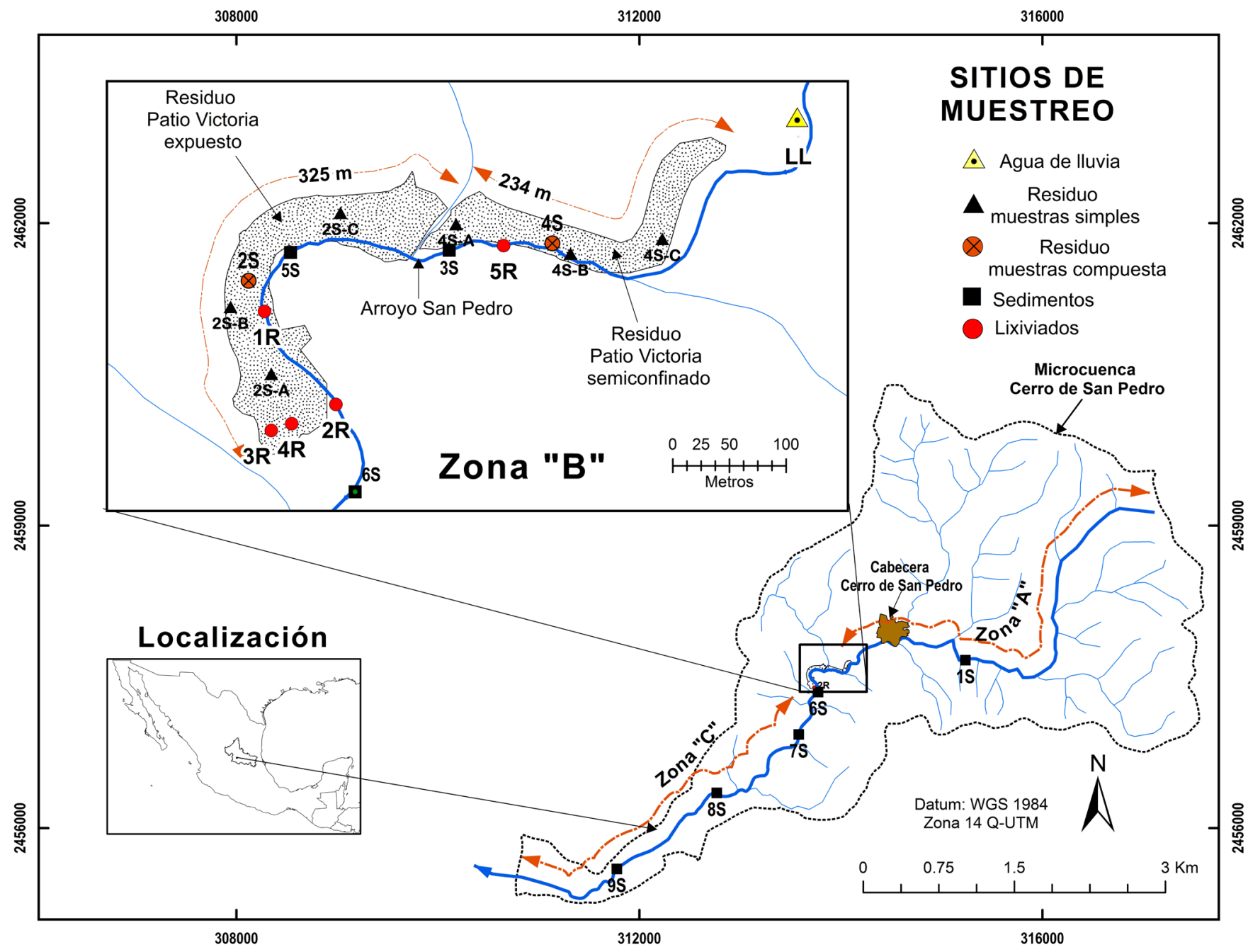

Figura 1 Localización del área de estudio.

que incluye: i) Formación Cenicera, ii) Pórfido San Pedro, iii) Andesita Casita Blanca, iv) Ignimbrita Santa María, v) Latita Portezuelo, vi) Ignimbrita Panalillo (Labarthe y Tristán, 1978). Asimismo, también se incluyen extensos depósitos de gravas del Pleistoceno. La mineralización de Cerro de San Pedro consiste en vetas, chimeneas y mantos presentes en las calizas adyacentes al pórfido (monzodiorítico) San Pedro. Así mismo este cuerpo presente una mineralización diseminada tipo stockwork. La zona superior de oxidación presenta una diseminación de $\mathrm{Au}-\mathrm{Ag}$ asociada con óxidos de Fe secundarios y es subyacida por una mineralización de sulfuros de $\mathrm{Au}-\mathrm{Ag}-\mathrm{Zn}$ y $\mathrm{Pb}$ (SGM, 2008).

\subsection{ORIGEN DE LOS RESIDUOS MINEROS HISTÓRICOS}

En la microcuenca Cerro San Pedro, a lo largo de más de 400 años han existido diferentes periodos de actividad minera, los principales elementos de interés económico del yacimiento mineral, han sido $\mathrm{Au}, \mathrm{Ag}, \mathrm{Zn}$ y $\mathrm{Pb}$; algunas de las principales bonanzas se desarrollaron en diferentes épocas 1592-1870, 1870-1895, 1895-1948 y 1948 a la fecha. El presente estudio se limita a los residuos mineros históricos Patio Victoria (PV), que provienen del beneficio de sulfuros (pirita, galena, arsenopirita, entre otros), y que estuvieron ubicados 
a lo largo de la margen derecha del arroyo San Pedro (actualmente el PV ha sido reubicado a un sitio controlado). Cuando se estudió, el PV tenía una longitud de $559 \mathrm{~m}$ y una altura promedio de $12 \mathrm{~m}$ (sobre el nivel del arroyo), está compuesto por material de granulometría heterogénea (grava a arenas finas). Por sus características en superficie y el tiempo desde que los residuos fueron depositados, el PV fue sido clasificado en dos zonas: i) semiconfinado (más de 350 años) y ii) expuesto $( \pm 70$ años) (Vázquez, 2012). Se diferencia de otros depósitos que existen en la subcuenca (terreros, escorias, calcinas, etc.) por el alto riesgo de movilidad de los elementos potencialmente tóxicos que contiene (Razo et al., 2007). El PV sobreyace a rocas sedimentarias calcáreas de la Formación La Peña, su mineralogía está constituida por: i) calcita, ii) cuarzo, iii) yeso, iv) muscovita, v) caolinita y vi) jarosita, además de sulfuros como pirita, arsenopirita, y galena principalmente (Martínez, 2015). Se ha estimado que contenía 150000 toneladas de residuos con un volumen de $120000 \mathrm{~m}^{3}$ (Razo et al., 2007), mediciones recientes señalan un volumen del orden $98000 \mathrm{~m}^{3}$ (Montes, 2016).

\section{Metodología}

\subsection{TOMA DE MUESTRAS Y MEDIGIONES DE GAMPO}

De acuerdo con estudios previos (Razo et al., 2007, Vázquez, 2012; Martínez, 2015) y observaciones de campo derivadas de la presente investigación, se ha identificado que el PV presentó generación de DAR. En base a esta condición, dimensiones del PV, zonas identificadas, y la NMX-AA-132SCFI-2006 (Secretaría de Economía, 2006) para la selección de los sitios de muestreo de residuos, se estableció un programa de muestreo usando el criterio a Juicio del experto. Para esta investigación, se tomó una muestra compuesta de residuo en cada una de las zonas del PV (muestra 2S que representa la zona expuesta y muestra $4 \mathrm{~S}$ que representa la zona confinada); cada una de estas muestras consideró un compósito de 3 muestras simples, obte- nidas de forma que fueran representativas de cada zona del PV (Figura 1). Cada muestra simple se obtuvo del material colectado en un canal $(15 \mathrm{~cm}$ ancho x $5 \mathrm{~cm}$ profundidad $\mathrm{x}$ largo variable entre 10-12 m) a lo largo de la pendiente en dirección al cauce. La cantidad total de muestra compuesta colectada (más de $100 \mathrm{~kg}$ ), se cuarteó en campo separando la fracción menor a $2 \mathrm{~mm}$, que posteriormente se homogenizó y cuarteó hasta reducir a $1 \mathrm{~kg}$ de muestra compuesta, que fue la porción que se consideró para los análisis.

A lo largo del tiempo, los residuos del PV han sido dispersados a lo largo del cauce del arroyo San Pedro por la erosión fluvial, generando modificación en la composición química de los sedimentos de arroyo. Se clasificaron 3 zonas para la toma de muestras de sedimento de arroyo: i) Zona A "aguas arriba" (control negativo), ii) zona B "intermedia" (impacto directo) y iii) zona C "aguas abajo", que representa el efecto del impacto ocasionado por la dispersión fluvial del material de PV, aunado a la dilución natural de los sustratos geológico y suelo en la microcuenca (Figura 1). Los sitios específicos en cada zona fueron seleccionados con criterio de muestreo dirigido (NMX-AA-132-SCFI-2006; Secretaría de Economía, 2006). La muestra de la zona A (1S) se colectó en el vaso de un bordo colector de agua, que presenta una importante acumulación de sedimentos captados del arroyo principal y un gran número de tributarios (Figura 1) se obtuvo en un área de $1 \mathrm{~m}^{2}$ hasta una profundidad de $5 \mathrm{~cm}$. En la zona B (muestras $3 \mathrm{~S}$ y $5 \mathrm{~S}$ ) se distribuyeron los puntos en forma equidistante a partir del inicio del PV, estas muestras representan el sedimento a lo largo de un canal transversal (10 $\mathrm{cm}$ de ancho x $5 \mathrm{~cm}$ profundidad) definido para la totalidad de la zona activa del cauce del arroyo. En la Zona C (muestras 6S, 7S, 8S, 9S) la distribución de los sitios de muestreo se estableció con base en las observaciones de campo, tomando la muestra (mismo procedimiento zona B) cuando se identificó presencia notoria en el cauce de sedimentos derivados del PV.

Para la colecta de agua de precipitación que tiene interacción con el PV y sedimentos de arroyo se 
estableció el siguiente criterio: i) tomar la muestra antes de contacto con el PV (agua de lluvia), y ii) después de contacto con el PV y acumulamiento sobre el sedimento de arroyo (lixiviado o DAR). Para colectar el agua de lluvia, se colocó una estación que incluye un recipiente de plástico $(20 \mathrm{~L}$ de capacidad) tomando la muestra después de cada evento de lluvia (LL) evitando que fuera afectada por la evaporación. Para la toma de muestras de DAR, mediante recorridos de campo se identificaron 3 sitios de acumulamiento de DAR después de los eventos de lluvia en cauce del arroyo: i) en la zona semi-confinada (muestra $5 \mathrm{R}$ ), ii) en la porción central de la zona expuesta (muestra $1 \mathrm{R}$ ) y iii) al final de la zona expuesta (muestra 2R). Otras dos muestras de DAR ( $3 R$ y $4 R$ ) se tomaron de acumulamientos identificados durante los recorridos de campo en la parte superior del depósito expuesto. La muestra 3R se tomó en una obra minera (cata o cueva) sobre la roca caliza, mientras que la $4 \mathrm{R}$ se tomó en zonas de acumulamiento de DAR producidos por bordos del mismo residuo. Para las muestras de lluvia y DAR, se midieron en campo parámetros como temperatura y $\mathrm{pH}$. Para las determinaciones de laboratorio se obtuvieron muestras filtradas $(0.45 \mu \mathrm{m})$ sin acidificar (aniones mayores) y acidificadas ( $\mathrm{pH} \sim 2$ ) (metales mayores, menores y traza, $\mathrm{NO}_{3}$ ), los frascos de polietileno de alta densidad se almacenaron a una temperatura de $4{ }^{\circ} \mathrm{C}$ para su transporte al laboratorio.

\subsection{ANÁLISIS DE LABORATORIO}

Con el fin de cuantificar la movilidad de PECT y MM, se realizó una prueba de extracción de acuerdo con los criterios establecidos en la NOM-141-SEMARNAT-2003 (Semarnat, 2004), que está basada en la prueba ASTM 3987-55. Consiste en lixiviar jales con agua desionizada y obtener una solución acuosa para analizar los compuestos lixiviados. La prueba considera que el agua de lluvia, baja en sales minerales, entra en contacto con un residuo minero localizado en una región árida. A las muestras de sedimentos y residuos (1S a 9S) se les practicaron extracciones con agua desionizada para determinar la fracción soluble. Después de que fueron homogeneizadas y cribadas, se pesó $1 \mathrm{~g}$ de muestra que se mezcló con $20 \mathrm{~mL}$ de agua desionizada en un tubo de fondo cónico, manteniéndolo en agitación por 18 horas en un agitador orbital. Después de la extracción se permitió la sedimentación de las muestras, determinando el pH del sobrenadante. Los extractos se filtraron empleando un filtro de $0.45 \mu \mathrm{m}$. La cuantificación de los cationes (Al, As, Ca, Fe, K, $\mathrm{Mg}, \mathrm{Mn}, \mathrm{Na}, \mathrm{Pb}, \mathrm{Si}, \mathrm{Zn}$ ) del extracto se determinó en el espectrofotómetro óptico ICP modelo iCAP 7400 Duo marca Thermo Scientific y los aniones $(\mathrm{Cl}$, $\mathrm{SO}_{4}$ ) por métodos analíticos Estándar de laboratorio. En el caso de la muestra de agua de lluvia y las 5 muestras de lixiviado la cuantificación de la concentración de especies acuosas se realizó en el espectrofotómetro ICP para cationes y por métodos Estándar de laboratorio para aniones. Las muestras de sedimento (1S), y residuo $(2 \mathrm{~S})$ y $(4 \mathrm{~S})$ se prepararon (cribado y homogeneización) para realizar análisis por difracción de polvo de rayos $\mathrm{X}$ en un equipo marca Bruker Do Advance.

Para el proceso de modelación se calculó tanto la evolución de las familias hidrogeoquímicas como el proceso de atenuación, empleando el código de computadora "Geochemist's Workbench" (GWB) versión 11.0 (Bethke, 2008). Para elaborar el diagrama de Piper se diseñó una hoja de cálculo con la aplicación GSS que se ligó al programa Gtplot; para calcular la saturación mineral $(\mathrm{Q} / \mathrm{K})$ de las muestras se empleó la aplicación $S p e c E$; los diagramas de Eh-pH se elaboraron con la aplicación Act2; el proceso de atenuación con calcita con la aplicación React, el de dilución de las muestras con agua de lluvia con la aplicación GSS y el de la evaporación por eliminación de agua y formación de fases minerales secundarias con la aplicación React. Las aplicaciones anteriores, GSS, specE8, Act2 y React, se ligaron a la aplicación Gtplot para la elaboración de las gráficas. 


\section{Resultados y discusión}

Los resultados muestran que existen diferencias significativas entre la mineralogía del sedimento de arroyo y los residuos mineros en TV (Figura 2). En todas las muestras se identificó el cuarzo, pero en la muestra de sedimento se encontraron calcita $\left(\mathrm{CaCO}_{3}\right)$, albita $\left(\mathrm{NaAlSi}_{3} \mathrm{O}_{8}\right)$ y montomorilonita $\mathrm{CaO}_{2}(\mathrm{Al}, \mathrm{Mg})_{2} \mathrm{Si}_{4} \mathrm{O}_{10}(\mathrm{OH})_{2} \cdot \mathrm{H}_{2} \mathrm{O}$, mientas que en los residuos se encontraron yeso $\left(\mathrm{CaSO}_{4} \cdot 2 \mathrm{H}_{2} \mathrm{O}\right)$ y jarosita $\mathrm{KFe}_{3}\left(\mathrm{SO}_{4}\right)_{2}(\mathrm{OH})_{6}$. Los resultados anteriores coinciden con los encontrados por Martínez (2015) y pueden estar indicando el efecto de atenuación natural por reacción de los minerales ricos en calcio, como la calcita y montomorilonita, para formar fases secundarias ricas en sulfatos como yeso y jarosita.

Es posible generar un drenaje ácido sintético, a partir de un residuo o sedimento, que se hace reaccionar con agua por un tiempo y relación sólido-solución definidos. La solución formada es el extracto acuoso que contiene las especies acuosas disueltas al final de la prueba. En los extractos acuosos obtenidos predominaron el Al, Ca, Fe, $\mathrm{SO}_{4}$ y Zn. Las concentraciones máximas de especies acuosas fueron mayores de la extracción de los residuos que la encontrada en los sedimentos: $\mathrm{Al}$ (34.47 mg/L vs $1.89 \mathrm{mg} / \mathrm{L}), \mathrm{Fe}(145 \mathrm{mg} / \mathrm{L}$ vs $0.29 \mathrm{mg} / \mathrm{L}), \mathrm{SO}_{4}(3900 \mathrm{mg} / \mathrm{L}$ os $1450 \mathrm{mg} / \mathrm{L}), \mathrm{Zn}$ $(51.41 \mathrm{mg} / \mathrm{L}$ os $0.23 \mathrm{mg} / \mathrm{L})$ probablemente debido a la presencia de yeso y jarosita en los residuos (Figura 2). A pH ácido (<4) el aluminio se mueve en el agua superficial, pero si el pH aumenta se hidroliza y se hace insoluble (Nordstrom et al., 2015). Este comportamiento de concentración de aluminio se observa en los residuos y los extractos de sedimentos de arroyo. Los resultados se muestran en la Tabla 1. Las fases minerales secundarias de las muestras sólidas, enriquecidas en hierro como la jarosita y en sulfatos como el yeso se disuelven más fácilmente que las fases primarias y como consecuencia el agua subterránea contiene altas concentraciones de $\mathrm{Fe}, \mathrm{SO}_{4}, \mathrm{Ca}, \mathrm{Na}, \mathrm{K}$ y Al. El $\mathrm{pH}(<2.7)$ de los extractos de los residuos mineros favoreció la disolución de las jarositas y explica la alta concentración de $\mathrm{SO}_{4}{ }^{2-}$ y Fe en estos extractos. La evolución de las familias hidrogeoquímicas, de bicarbonatada cálcica hacia sulfatada cálcica, se muestra en el diagrama Piper (Figura 3), que es probablemente debido al efecto de ión común, que permite que la calcita se haya precipitado y el yeso disuelto; ambas fases minerales contienen calcio, siendo el yeso más soluble que la calcita. En los lixiviados mineros, tomados de la superficie de los charcos frente a los residuos de TV, predominan las especies de $\mathrm{Al}, \mathrm{Ca}, \mathrm{Fe}, \mathrm{SO}_{4}$ y $\mathrm{Zn}$ de manera similar a como ocurre con los extractos acuosos de laboratorio. Las concentraciones máximas de especies acuosas fueron mayores de los charcos localizados enfrente de los residuos expuestos que del charco enfrente de los residuos confinados: Al (499.01 mg/L vs $402.97 \mathrm{mg} / \mathrm{L}), \mathrm{Ca}(629.05 \mathrm{mg} / \mathrm{L}$ vs $552.75 \mathrm{mg} / \mathrm{L})$, Fe $(9128.57 \mathrm{mg} / \mathrm{L}$ vs 3008.58 $\mathrm{mg} / \mathrm{L}), \mathrm{SO}_{4}(28271.34$ vs $14457.39 \mathrm{mg} / \mathrm{L})$, y $\mathrm{Zn}$ (2086.06 mg/L vs $1287.51 \mathrm{mg} / \mathrm{L}$ ). El haber confinado los residuos con el muro de mampostería permitió controlar mejor no solo la disolución sino la dispersión de los residuos mineros conducidos por el agua superficial del arroyo San Pedro. En un modelo de interacción residuo-agua el agua de lluvia disuelve parcialmente a los minerales que estos contienen y los incorpora al agua que escurre en el arroyo. Los resultados del análisis de muestras de agua de lluvia y lixiviados se muestran en la Tabla 2. Nordstrom et al. (2015) emplean el término drenaje ácido de roca (DAR) para referirse de forma general a un drenaje con las características de este estudio.

\section{Proceso de modelación}

\subsection{COMPARAGIÓN HIDROGEOQUÍMICA ENTRE EXTRACTOS ACUOSOS Y LIXIVIADOS}

Las concentraciones de $\mathrm{SO}_{4}, \mathrm{As}, \mathrm{Zn}, \mathrm{Ca}$ y Fe de los extractos acuosos se compararon con los obtenidos en los lixiviados. Los análisis de los lixiviados muestran altas concentraciones de sulfatos $(>$ $5000 \mathrm{mg} / \mathrm{L}$ ) y calcio (> $440 \mathrm{mg} / \mathrm{L})$ así como pH ácido $(<3)$ (Tabla 2); los análisis de los extractos 


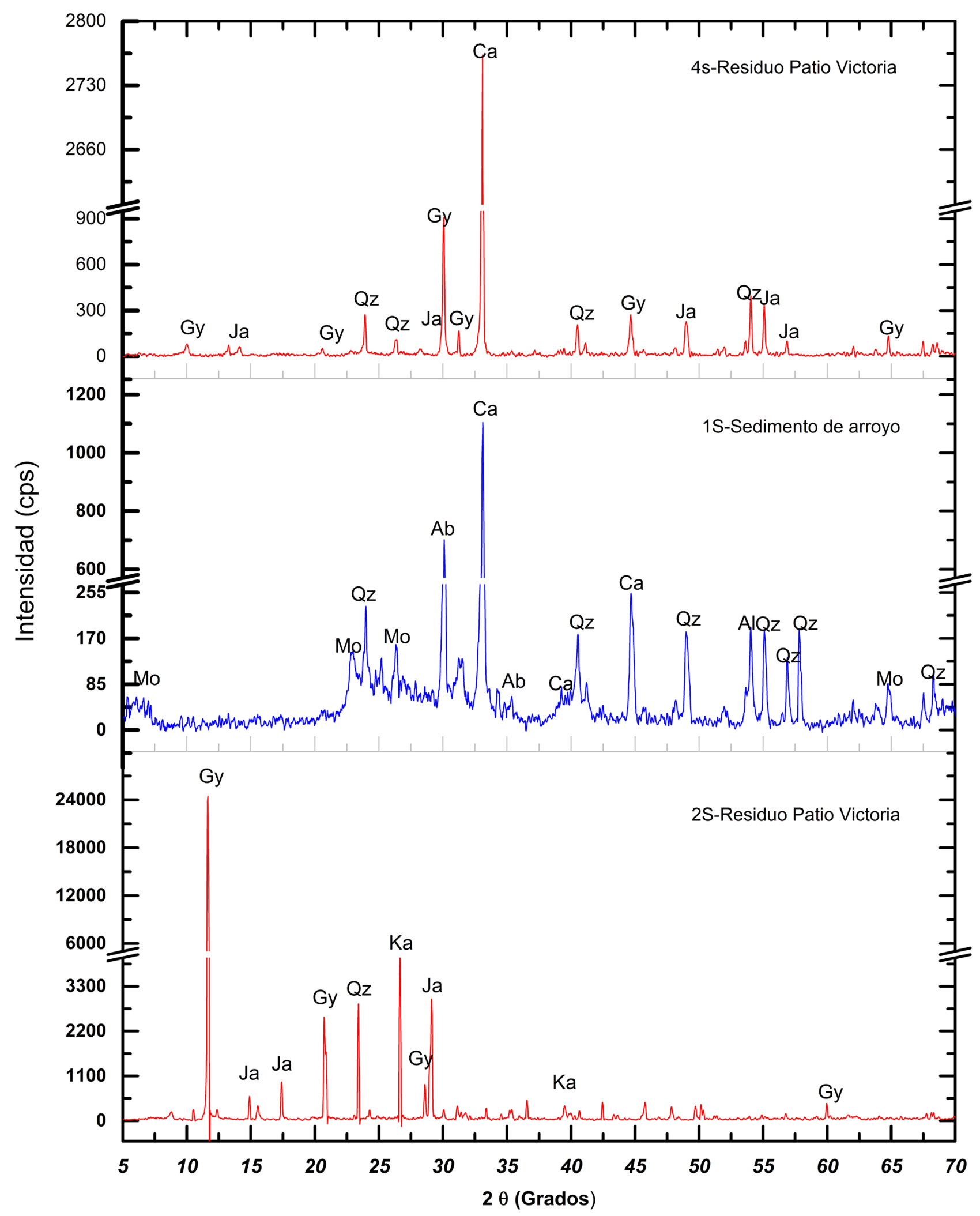

Figura 2 Difractogramas del sedimento de arroyo (1S) y de los residuos mineros Patio Victoria (2S) y (4S). Gy=Yeso, Qz= Cuarzo, $\mathrm{Ca}=$ Calcita, $\mathrm{Ab}=\mathrm{Albita}$, Ja=Jarosita, Mo= Montmorillonita, Ka=Caolinita. Modificado de Aragón (2016). 
Tabla 1. Concentración de especies acuosas ( $\mathrm{mg} / \mathrm{L}$ ) de los resultados de la Prueba de movilidad con agua meteórica en muestras de sedimentos de arroyo (1S, 3S, 5S, 6S, 7S, $8 \mathrm{~S}, 9 \mathrm{~S})$ y residuos mineros $(2 \mathrm{~S}, 4 \mathrm{~S})$.

\begin{tabular}{|c|c|c|c|c|c|c|c|c|c|}
\hline ID & $1 S$ & $2 S$ & $3 \mathrm{~S}$ & $4 S$ & 5S & $6 S$ & $7 \mathrm{~S}$ & $8 S$ & 9S \\
\hline $\mathrm{pH}$ & 7.46 & 2.55 & 8.13 & 2.7 & 7.19 & 7.85 & 8.04 & 8.14 & 8.34 \\
\hline $\mathrm{Al}$ & 0.60 & 34.47 & 1.89 & 31.17 & 0 & 0 & 0.19 & 0.32 & 0.81 \\
\hline As & 0.02 & 0.51 & 0.01 & 0.19 & 0 & 0 & 0 & 0 & 0 \\
\hline $\mathrm{Ca}$ & 21.84 & 238.9 & 9.42 & 237.3 & 257.2 & 45.22 & 21.38 & 10.87 & 10.08 \\
\hline $\mathrm{Cu}$ & 0.01 & 0.48 & 0.01 & 0.29 & 0 & 0 & 0 & 0 & 0.003 \\
\hline $\mathrm{Fe}$ & 0.08 & 145 & 0.29 & 48.77 & 0.19 & 0.04 & 0.04 & 0.09 & 0.18 \\
\hline $\mathrm{Mg}$ & 0.40 & 6.67 & 0.30 & 3.34 & 8.81 & 0.59 & 0.23 & 0.15 & 0.16 \\
\hline $\mathrm{Mn}$ & 0.009 & 1.83 & 0.02 & 3.3 & 0.04 & 0.01 & 0.01 & 0.02 & 0.05 \\
\hline $\mathrm{Pb}$ & 0 & 0.06 & 0 & 0.22 & 0 & 0 & 0 & 0 & 0.05 \\
\hline $\mathrm{SO}_{4}$ & 135 & 3900 & 45 & 1700 & 1450 & 162.5 & 45 & 245 & 27 \\
\hline $\mathrm{Si}$ & 4.37 & 0.22 & 3.31 & 0.26 & 0.72 & 0.92 & 1.16 & 1.04 & 1.31 \\
\hline $\mathrm{Zn}$ & 0.02 & 51.41 & 0.12 & 28.79 & 0.14 & 0.04 & 0.041 & 0.11 & 0.23 \\
\hline $\mathrm{Cl}$ & 4.82 & 2230 & 4.82 & 301.35 & 7.23 & 36.16 & 9.64 & 24.11 & 4.82 \\
\hline $\mathrm{K}$ & 3.04 & 0 & 1.03 & 0 & 0.72 & 1.53 & 0.9 & 0.72 & 0.84 \\
\hline $\mathrm{Na}$ & 0.91 & 0.001 & 0.71 & 0.001 & 0.001 & 1.08 & 0.001 & 0.001 & 0.001 \\
\hline $\mathrm{HCO}_{3}$ & 65.44 & 0 & 76.35 & 0 & 76.35 & 54.53 & 76.35 & 141.8 & 65.44 \\
\hline
\end{tabular}

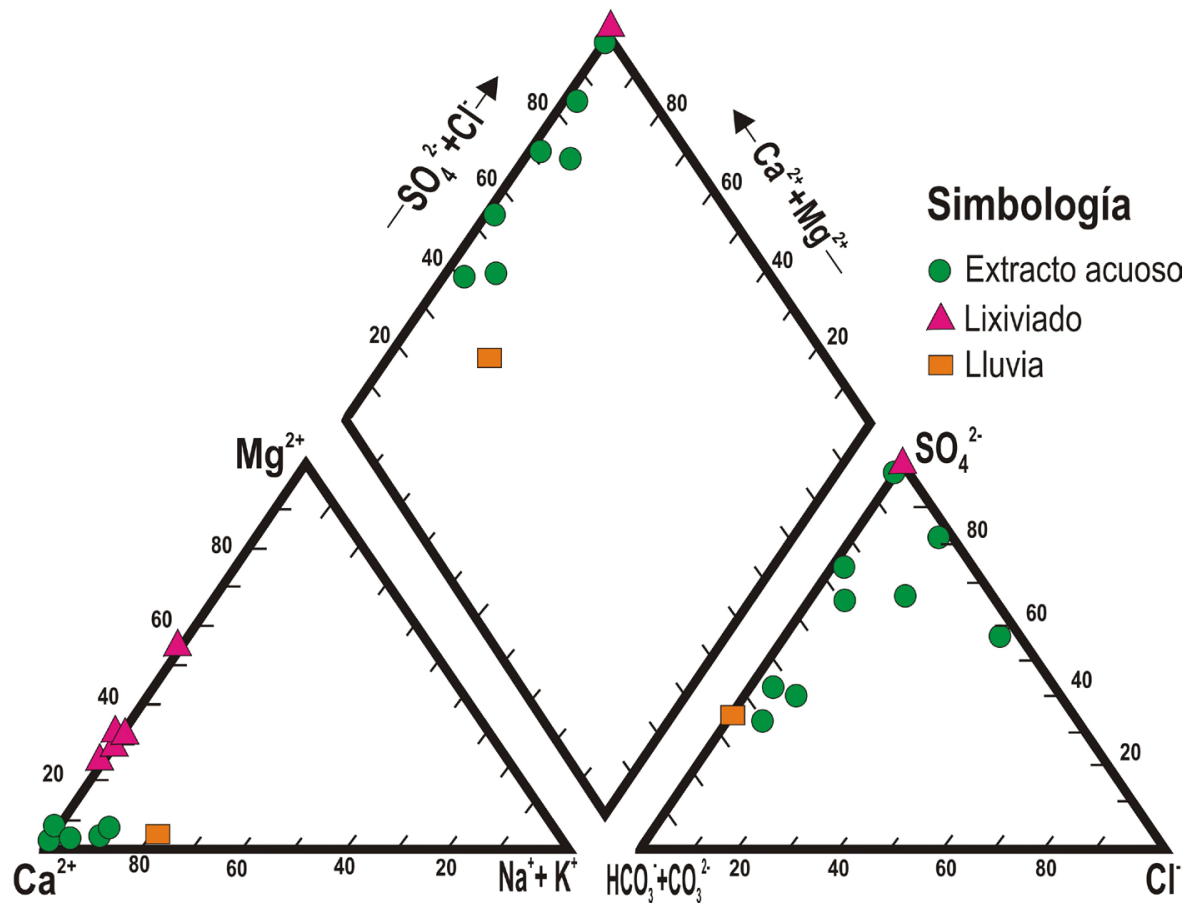

Figura 3 Diagrama de Piper para las muestras de extracto acuoso, lluvia y lixiviado. 
Tabla 2. Concentración de especies acuosas (mg/L) de muestras de agua de lluvia (LL) y de lixiviados (R).

\begin{tabular}{|l|r|r|r|r|r|r|}
\hline \multicolumn{1}{|c|}{$\mathbf{I D}$} & $\mathbf{L L}$ & \multicolumn{1}{c|}{$\mathbf{1 R}$} & \multicolumn{1}{c|}{ 2R } & \multicolumn{1}{c|}{ 3R } & \multicolumn{1}{c|}{ 4R } & \multicolumn{1}{c|}{ 5R } \\
\hline $\mathrm{pH}$ & 7.03 & 2.17 & 2.4 & 2.55 & 2.32 & 2.32 \\
\hline $\mathrm{Al}$ & 0.09 & 340.59 & 130.07 & 157.18 & 499.01 & 402.97 \\
\hline $\mathrm{As}$ & 0.02 & 19.92 & 7.08 & 6.05 & 123.67 & 45.67 \\
\hline $\mathrm{Ca}$ & 19.58 & 580.17 & 441.64 & 570.44 & 629.05 & 552.76 \\
\hline $\mathrm{Cu}$ & 0.02 & 9.21 & 4.36 & 4.66 & 15.35 & 10.60 \\
\hline $\mathrm{Fe}$ & 0.21 & 2236 & 786 & 820 & 9129 & 3009 \\
\hline $\mathrm{Mg}$ & 0.48 & 364.19 & 116.06 & 140.82 & 108.47 & 115.79 \\
\hline $\mathrm{Mn}$ & 0.005 & 144.85 & 41.97 & 44.24 & 45.21 & 74.88 \\
\hline $\mathrm{Pb}$ & 0.004 & 0.47 & 0.38 & 0.75 & 1.81 & 1.89 \\
\hline $\mathrm{SO}$ & 21.89 & 15035 & 5311 & 8126 & 28271 & 14457 \\
\hline $\mathrm{Si}$ & 2.28 & 6.35 & 5.81 & 2.92 & 1.85 & 4.97 \\
$\mathrm{Zn}$ & 0.27 & 2086.06 & 612.28 & 687.04 & 1264.42 & 1287.51 \\
\hline $\mathrm{K}$ & 2.23 & 6.85 & 4.49 & 0.43 & 0.23 & 3.71 \\
\hline $\mathrm{Na}$ & 4.71 & 6.79 & 4.27 & 0.70 & 0.21 & 4.11 \\
\hline
\end{tabular}

acuosos de los residuos también muestran altas concentraciones de sulfatos $(>1700 \mathrm{mg} / \mathrm{L})$ y calcio $(>230 \mathrm{mg} / \mathrm{L})$ y pH ácido $(<3)$; sin embargo, las concentraciones de los extractos acuosos de los sedimentos de arroyo en sulfatos $(<165 \mathrm{mg} / \mathrm{L})$ y calcio $(<250 \mathrm{mg} / \mathrm{L})$ son menores que las de los residuos y lixiviados. Los extractos acuosos más contaminados son los de los residuos mineros que se corresponden espacialmente con los lixiviados, reflejando la capacidad limitada de los sedimentos de arroyo para retener metales. (Figura 1; Tabla 1 y 2). El rango de concentración de arsénico para extractos de residuos y lixiviados $(0.19-123$ $\mathrm{mg} / \mathrm{L}$ ) es mucho mayor que para sedimentos (< $0.02 \mathrm{mg} / \mathrm{L}$ ); las concentraciones de zinc para extractos de residuos y lixiviados (> $20 \mathrm{mg} / \mathrm{L}$ ) son superiores que las encontradas en los extractos de sedimentos $(<0.2 \mathrm{mg} / \mathrm{L})$. Estos resultados muestran claramente que el zinc y arsénico en el agua proviene de la disolución de los residuos (Tablas 1-2). Las diferencias entre extractos acuosos y lixiviados se deben a las diferentes condiciones de muestreo y los mecanismos de disolución de las especies acuosas. Las familias hidrogeoquímicas de los extractos evolucionaron de tipo $\mathrm{HCO}_{3}-\mathrm{Ca}$ hacia $\mathrm{SO}_{4}$-Ca, de acuerdo al diagrama Piper (Figura 3), probablemente debido al efecto de ión común; la calcita es menos soluble que el yeso y la disolución de yeso aporta grandes cantidades de $\mathrm{Ca}^{+2}$ lo que fue rza la precipitación de calcita y la reducción de la concentración de bicarbonatos (Šráček et al., 2013).

\subsection{GÁLGULOS TERMODINÁMICOS}

En la Figura 4 se identifican las muestras de extracto acuoso de sedimento $(1 \mathrm{~S}, 3 \mathrm{~S}, 4 \mathrm{~S}, 5 \mathrm{~S}, 6 \mathrm{~S}$ y $7 \mathrm{~S})$, extracto acuoso de residuo ( $2 \mathrm{~S}$ y $4 \mathrm{~S})$ y lixiviados (\#R). La saturación mineral $(\mathrm{Q} / \mathrm{K})$ para estos tres grupos de sistemas acuosos en cuanto a yeso $\mathrm{CaSO}_{4} \cdot 2 \mathrm{H}_{2} \mathrm{O}$ fue $0.0039,0.08$ y 1 (en equilibrio); anglesita $\mathrm{PbSO}_{4}$ : ausente, 0.06 y $>1$ (en equilibrio), calcita $\mathrm{CaCO}_{3}:<1$ (en equilibrio), ausente, arseniatos metálicos $\mathrm{M}^{+}\left(\mathrm{AsO}_{4}\right)_{2}(\mathrm{c})$ : ausente, $>10$. Las muestras que están subsaturadas, $(\mathrm{Q} / \mathrm{K})<1$, se están disolviendo, las que están sobresaturadas 


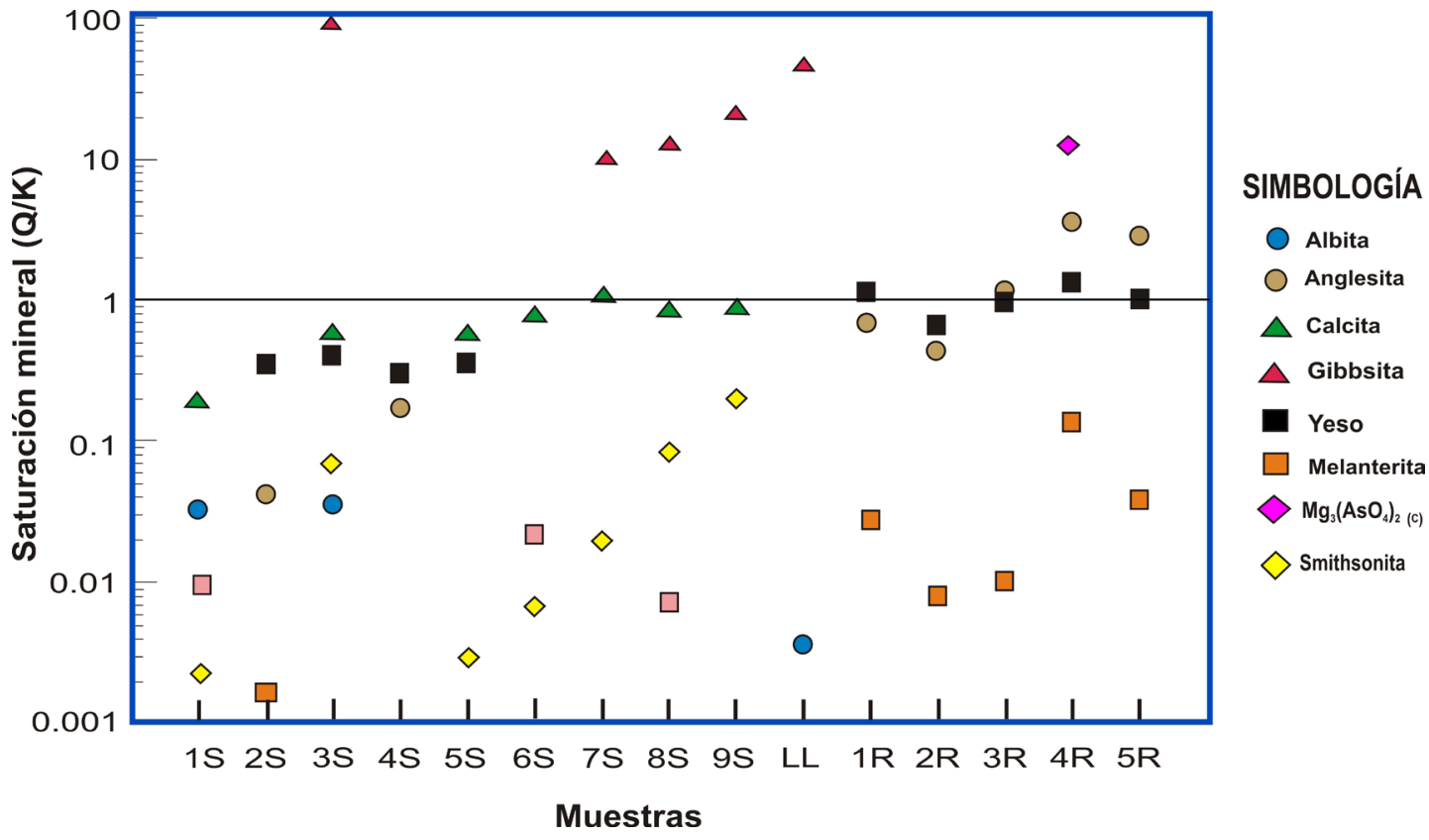

Figura 4 Saturación mineral (Q/K) para las muestras de extracto acuoso de residuo, lluvia y lixiviado.

$(\mathrm{Q} / \mathrm{K})>1$ se están precipitando y las que están en equilibrio tienen un $(\mathrm{Q} / \mathrm{K})=1$. Los resultados anteriores indican que el yeso en los lixiviados está sobresaturado y se confirma con la presencia de yeso detectada por DRX de los residuos que provienen del mismo sitio; la saturación de yeso controla la concentración de sulfatos en el agua (Romero et al., 2007; Huisamen y Wolkersdorfer, 2016). Los procesos de disolución y precipitación de fases minerales en cada sistema acuoso son completamente diferentes; en el extracto acuoso de sedimento de arroyo se disuelve calcita y en menor proporción yeso, en el extracto acuoso de residuo se disuelven sulfatos, principalmente de calcio y plomo, mientras que en el lixiviado se alcanzan el equilibrio de disolución-precipitación de los sulfatos de calcio y plomo, se disuelven los sulfatos de hierro y se precipitan los arseniatos metálicos. Los resultados anteriores son comparables con los reportados por Sánchez-España y Diez-Ercilla (2008) y Frau et al. (2011).

En la Figura 5 se muestran los diagramas de Eh-pH para el extracto acuoso del residuo expuesto $2 \mathrm{~S}$ (Figura 5.a) y el lixiviado 4R (Figura 5.b). De acuerdo al $\mathrm{pH}$ de estas muestras (línea vertical para pH 2.3 y 2.5) las especies acuosas predominantes son sulfatos, y las fases minerales son: jarosita, realgar, esfalerita y arsenopirita. El Eh es variable y el pH fijo, marcado con líneas verticales, de 2.55 y $2.33,2 \mathrm{~S}$ y $4 \mathrm{R}$, respectivamente. Los logaritmos de actividad para $2 \mathrm{~S}$ y $4 \mathrm{R}$ son: $\mathrm{SO}_{4}(-1.518$ y -1.954$)$, $\mathrm{Fe}^{++}(-1.707$ y -3.231$), \mathrm{Zn}^{++}(-2.737$ y -3.828$)$, $\mathrm{H}_{2} \mathrm{AsO}_{4}-(-3.828$ y -5.681$), \mathrm{Ca}^{++}(-2.795$ y -2.935$)$, respectivamente. En ambos gráficos, las zonas de estabilidad de las fases acuosas de sulfatos ocurren principalmente para $\mathrm{pH}$ ácido-neutro (entre $0 \mathrm{y}$ 10) y condición oxidante ( $\mathrm{Eh} \approx-0.30$ a 1.1 volts). $\mathrm{La}$ actividad de $\mathrm{Fe}$ es mayor en los lixiviados que en los extractos acuosos de residuos, el exceso de Fe disuelto corresponde a la coloración roja ocre de los lixiviados, e indica que la fase acuosa en el sistema es $\mathrm{FeSO}_{4}$. El pH del agua ácido y una condición oxidante favorecen la precipitación de jarosita. Los resultados están de acuerdo con Quispe et al., 2013.

\subsection{PROGESO DE ATENUAGIÓN}

\subsubsection{ATENUACIÓN CON CALCITA}

Tanto el extracto acuoso de residuo (2S) como el lixiviado (4R), ambos cercanos espacialmente entre sí, reacción con $4 \mathrm{~g}$ de calcita, cantidad pro- 

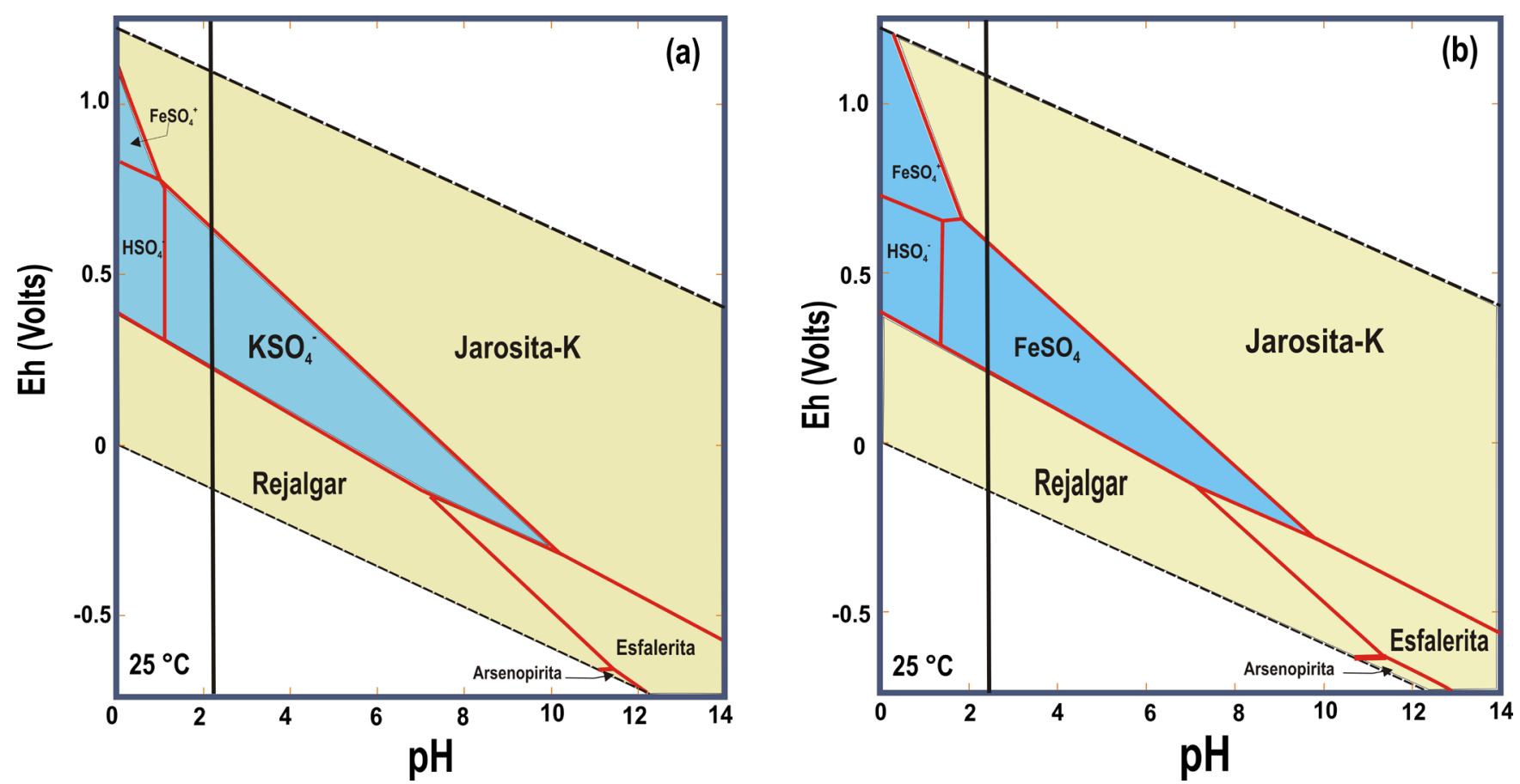

Figura 5 Diagramas de Eh-pH de extracto acuoso del residuo $2 \mathrm{~S}$ (a) y del lixiviado 4R (b). El sistema S-Fe- $\mathrm{Zn}-\mathrm{As}-\mathrm{K}-\mathrm{Ca}-\mathrm{Na}-\mathrm{H}_{2} \mathrm{O}$ asume una temperatura de $25^{\circ} \mathrm{C}$ y $1 \mathrm{~atm}$ de presión. Se muestran los campos de influencia de los sulfatos metálicos en fase acuosa (azul) y de las jarositas (café) en fase sólida para condiciones oxidantes (Eh $>0$ ).

puesta en el patrón de reacción, incrementando el pH del sistema acuoso, como se observa en la Figura 6. Para la muestra 2S (Figura 6, a y b) el incremento fue de 2.5 a 8.7 y para la muestra $4 \mathrm{R}$ (Figura 6, с y d) de 2.3 a 6.2. Los cambios de $\mathrm{pH}$ coinciden con la precipitación de las fases minerales secundarias, principalmente hidróxidos metálicos, que se muestran en los gráficos de saturación mineral $(\mathrm{Q} / \mathrm{K})$. Este comportamiento coincide con el propuesto por Šráček et al. (2013) y Corrales-Pérez y Romero, (2013). Así mismo, se observan las fases minerales sobresaturadas $(\mathrm{Q} / \mathrm{K})>1$ que precipitan en al agua como gibbsita $\mathrm{Al}(\mathrm{OH})_{3}$ y alunita $\mathrm{K} \mathrm{Al}_{3}(\mathrm{OH})_{6}\left(\mathrm{SO}_{4}\right)_{2}$, smithsonita $\mathrm{ZnCO}_{3}$ y arseniatos de zinc $\mathrm{Zn}_{3}\left(\mathrm{AsO}_{4}\right)_{2}$. Bajo condiciones alcalinas la goethita se vuelve la fase secundaria predominante (Nordstrom et al., 2015). En el lixiviado se precipitan a la vez carbonatos, arseniatos, sulfatos e hidróxidos metálicos. La precipitación de alunita $\mathrm{K} \mathrm{Al}_{3}(\mathrm{OH})_{6}\left(\mathrm{SO}_{4}\right)_{2}$ en el agua es consecuencia de la disolución de la anglesita $\mathrm{PbSO}_{4}$. Bajo condiciones de $\mathrm{pH}$ ácido (2.5), la jarosita $\mathrm{K}$ $\mathrm{Fe}_{3}\left(\mathrm{SO}_{4}\right)_{2}(\mathrm{OH})_{6}$ es un mineral estable capaz de inmovilizar algunos de los metales liberados en el lixiviado. En condiciones de $\mathrm{pH}$ mayores (> 2.5) la jarosita se vuelve inestable y libera metales al agua (Gasparon et al., 2007) en forma de arseniatos de Ca, Zn y Mn (Figura 6).

\subsubsection{ATENUACIÓN CON AGUA DE LLUVIA}

En el modelo de atenuación el agua de lluvia $(\mathrm{pH}$ 7) se mezcla primero con el extracto acuoso de residuo $(2 \mathrm{~S})$ y después con el lixiviado (4R). La solución-mezcla incrementa gradualmente su $\mathrm{pH}$, en función de la relación de mezcla (Figura 7); El $\mathrm{pH}$ inicial de ambos sistemas acuosos fue de $2.5 \mathrm{y}$ alcanza un valor de 6 y 4.9 cuando la relación es 1:1000. Este comportamiento demuestra el efecto de dilución con agua de lluvia. De manera similar se puede producir un efecto de atenuación natural de un lixiviado con agua de río (Berger et al., 2000).

\subsubsection{ATENUACIÓN POR EVAPORACIÓN}

Las muestras de extracto acuoso de residuo (2S) y lixiviado (4R) siguiendo un patrón de evaporación, redujeron su volumen de agua de 1000 a 

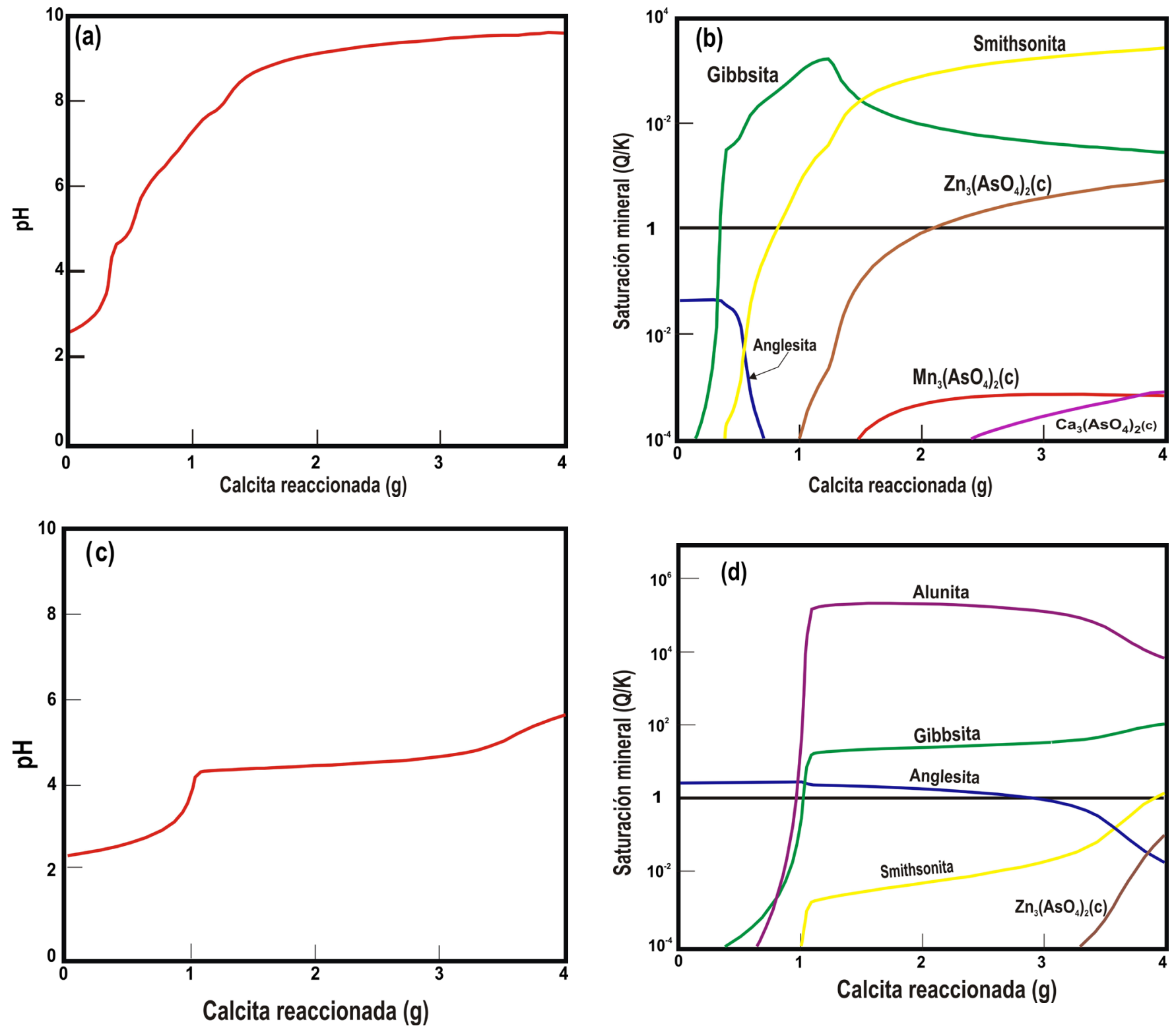

Figura 6 pH del agua y solubilidad de los minerales (Q/K), para extracto acuoso de residuo $2 \mathrm{~S}$ (a y b) y del lixiviado 4R (c y d), en función de la cantidad de calcita ( $g$ ).

1 gramo y forzaron la precipitación de fases minerales secundarias (Figura 8). En el proceso de evaporación se precipitan diversas fases minerales, para el extracto (2S) las fases minerales fueron yeso $\left(\mathrm{CaSO}_{4}: 2 \mathrm{H}_{2} \mathrm{O}\right)$, anglesita $\left(\mathrm{PbSO}_{4}\right)$ y cuarzo $\left(\mathrm{SiO}_{2}\right)$ mientras que para el lixiviado se obtuvo melanterita $\left(\mathrm{FeSO}_{4}: 7 \mathrm{H}_{2} \mathrm{O}\right)$, epsomita $\left(\mathrm{MgSO}_{4}: 7 \mathrm{H}_{2} \mathrm{O}\right)$, yeso $\left(\mathrm{CaSO}_{4}: 2 \mathrm{H}_{2} \mathrm{O}\right)$, cuarzo $\left(\mathrm{SiO}_{2}\right)$, mercalita $\left(\mathrm{KHSO}_{4}\right)$ y anglesita $\left(\mathrm{PbSO}_{4}\right)$. La variedad y cantidad de fases minerales precipitadas es 2 órdenes de mag- nitud mayor en el lixiviado que en el extracto acuoso de residuo. Cuando la oxidación de sulfuros se combina con evaporación puede generar un drenaje excesivamente ácido con altísimas concentraciones de sustancias disueltas que precipitan cuando la muestra acuosa pierde agua. La precipitación de fases secundarias de melanterita puede estar favoreciendo la atenuación natural de $\mathrm{Pb}$ en los lixiviados de la mina (Romero et al., 2007; Lindsay et al., 2015). La formación de fases mine- 
rales secundarias de Fe y de sulfato como jarosita, yeso y melanterita, se han observado en materiales de relleno en minas a cielo abierto bajo condiciones oxidantes (Huisamen y Wolkersdorfer, 2016). En los recorridos de campo se observan en el área de estudio, especialmente en épocas de intenso calor, costras de residuos (hardpans) que se forman por procesos de evaporación (Meima et al., 2007) y en los mismos sitios la presencia de charcos de lixiviados con la típica coloración ocre de los drenajes

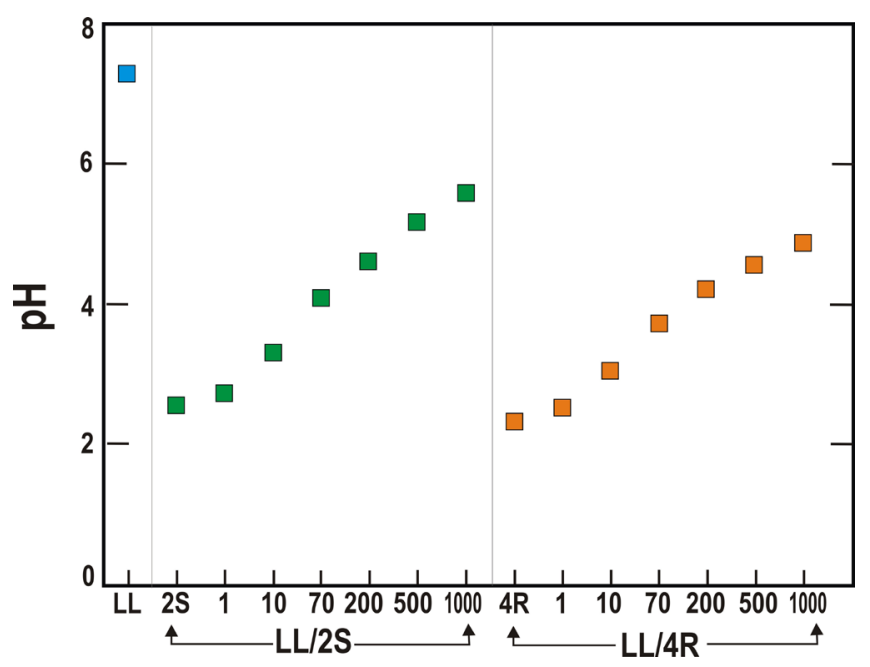

Figura 7 Aumento en el pH al cambiar la relación de la mezcla agua de Iluvia-extracto acuoso (LL/2S) o lluvia-lixiviado (LL/4R).

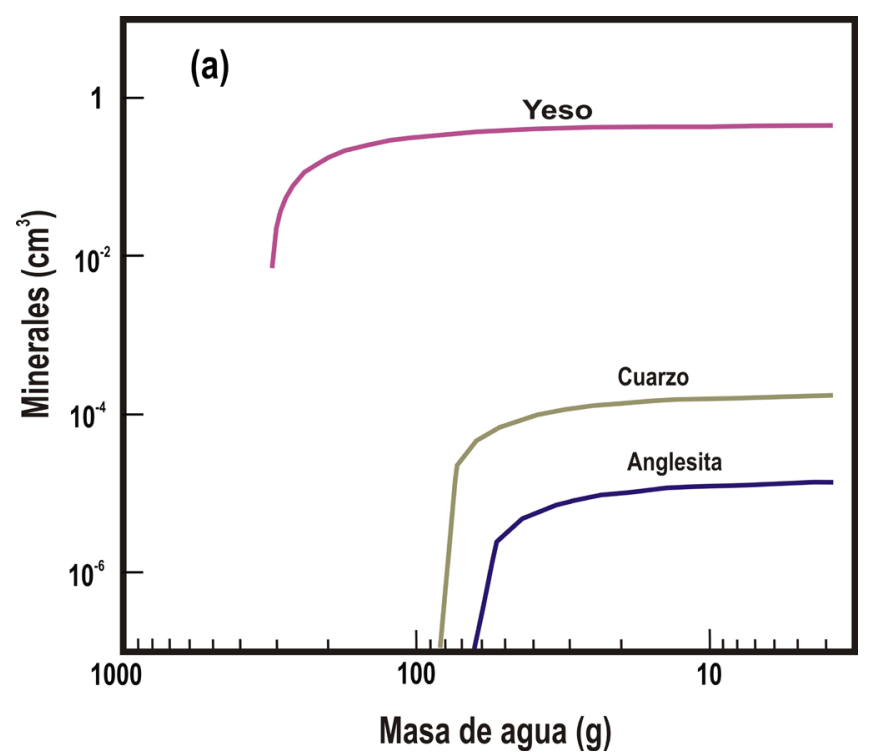

ácido de mina en épocas de lluvia.

\section{Conclusiones}

Las concentraciones de especies acuosas de $\mathrm{Al}$, $\mathrm{Ca}, \mathrm{Fe}, \mathrm{SO}_{4}$, As y $\mathrm{Zn}$ fueron mayores en los lixiviados de los residuos históricos Terreros Victoria (TV), localizados al sureste del poblado de Cerro de San Pedro en el estado de San Luis Potosí, que en los extractos acuosos, producidos mediante la prueba de extracción de elementos potencialmente tóxicos (EPTs), de los residuos y sedimentos del mismo sitio. Los sedimentos de arroyo de Cerro de San Pedro contienen cuarzo, calcita, albita y montomorilonita, mientas que los residuos presentan cuarzo, yeso y jarosita. La evolución del agua, de bicarbonatada cálcica en el extracto acuoso de sedimento a sulfatada cálcica en los residuos, probablemente se deba al efecto de ión común. Mientras que el extracto acuoso de sedimento de arroyo se disuelve calcita en el lixiviado se disuelven sulfatos de hierro y se precipitan los arseniatos metálicos. La reacción con calcita, mezcla con agua de lluvia y evaporación atenuaron exitosamente estos lixiviados. Con la presencia de calcita se disuelven y/o precipitan las fases minerales de $\mathrm{Al}$ como

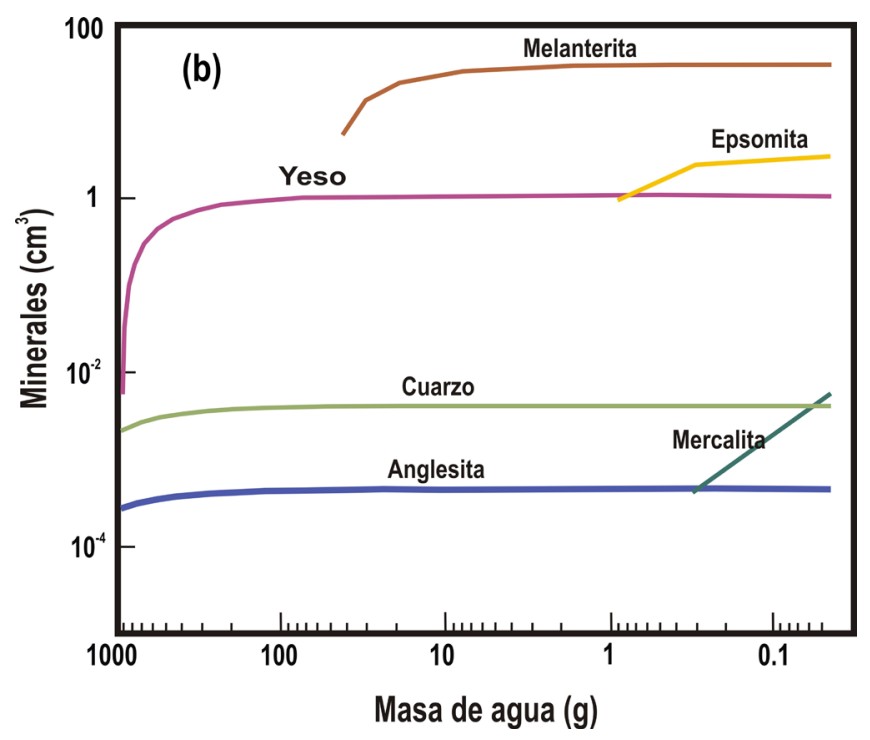

Figura 8 Precipitación de fases minerales en el extracto acuoso de residuo $2 \mathrm{~S}$ (a) y en el lixiviado $4 \mathrm{R}$ (b) al evaporarse una masa de $1000 \mathrm{~g}$ de agua. 
la gibbsita, de $\mathrm{Pb}$ como la anglesita y de sulfato como el yeso y la melanterita. La atenuación por reacción con calcita, y por evaporación resultó ser mayor en los lixiviados que en los extractos acuosos de residuos o sedimentos. Los estudios de modelación hidrogeoquímica generan posibles escenarios de atenuación que complementan la prueba de extracción de residuos mineros de la NOM-141-SEMARNAT-2003.

\section{Agradecimientos}

Los autores agradecen a las revisoras del artículo, Dra. Pura Alfonso y anónima, los comentarios y observaciones que permitieron mejorarlo sustancialmente. El presente proyecto contó con el apoyo económico del fondo Fordecyt clave 190966. Se agradece al Dr. Erik Espinosa, a la Dra. María Elena García y a la Maestra Ana María Guadalupe López Hernández, del laboratorio de análisis de la Facultad de Ingeniería y del Laboratorio de Geoquímica del Instituto de Geología UASLP, por su valioso apoyo en el análisis de las muestras de extractos acuosos y lixiviados. Se agradece a Sócrates Alonso, del Área de Ciencias de la Tierra de la Facultad de Ingeniería UASLP, por su valioso apoyo en las actividades de muestreo en campo y elaboración de mapas.

\section{Referencias}

Amos, R.T., Blowes, D.W., Bailey, B.L., Sego, D.C., Smith, L., Ritchie, A.L., 2015, Wasterock hydrogeology and geochemistry: Applied Geochemistry, 57, 140-156.

Aragón, G.G., 2016, Caracterización mineralógica de residuos mineros de los distritos de Cerro de San Pedro y Santa María de la Paz, San Luis Potosí, Universidad Autónoma

de San Luis Potosí, Trabajo recepcional, Facultad de Ingeniería, 115 p.

Aranda-Gómez, J.J., 1989, Geología preliminar del graben de Aguascalientes, Universidad
Nacional Autónoma de México: Revista del Instituto de Geología, 8, 22-32.

Armienta, M.A., Segovia N., 2008, Arsenic and Fluoride in the groundwater of Mexico: Environ Geochem Health, 30, 345-353.

Berger, A.C., Bethke, C.M., Krumhansl, J.L., 2000, A process model of natural attenuation in drainage from a historic mining district: Applied Geochemistry, 15, 655-666.

Bethke, C.M., 2008, Acid drainage, Geochemical and biogeochemical reaction modeling, University of Illinois, Urbana-Champaign, Cambridge University Press, 449-456.

Cardona, A., Carrillo-Rivera, J.J., CastroLarragoitia, G.J., Graniel-Castro, E.H., 2008, Combined use of indicators to evaluate waste-water contamination to local flow systems in semi-arid regions: San Luis Potosi, Mexico: Selected Papers XXXIII-IAH Internacional Congress, Zacatecas, Mexico. Asociación Internacional Hidrogeólogos, Ed Balkema, Taylor \& Francis Group. Leiden, The Netherlands, 12, 85-104.

Carrillo-Chávez, A., Salas-Megchún, E., Levresse, G., Muñoz-Torres, G., Pérez-Arvizu, O., Gerke, T., 2014, Geochemistry and mineralogy of mine-waste mineral from a "skarn-type" deposit in central Mexico, Modeling geochemical controls of metals in the Surface environment: Journal of Geochemical Exploration, 144A, 28-36.

Castro-Larragoitia, J., Kramar, U., Puchelt, H., 1997, 200 years of mining activities at La Paz/ San Luis Potosi/Mexico - Consequences for environment and geochemical exploration: Journal of Geochemical Exploration, 58, 81-91.

Corrales-Pérez, D., 2014, Evaluación de diferentes materiales Geológicos para el tratamiento pasivo del Drenaje ácido de minas, en TaxcoGuerrero: Universidad Nacional Autónoma de México, Tesis de doctorado del Posgrado Ciencias de la tierra, Instituto de Geología.

Corrales-Pérez, D., Romero, F.M., 2013, Evaluación de la peligrosidad de jales de 
zonas mineras de Nicaragua y México y alternativas de solución: Boletín de la Sociedad Geológica Mexicana, 65(3), 427-446.

Frau, F., Cidu, R., Dadea, C., 2011, AMD generation from dissolution of secondary metal sulphates, in International Mine Water Association, Aachen, Germany "Mine Water - Managing the Challenges", 301-306.

Gasparon, M., Smedley, A., Jong, T., Costagliola, P., Benvenuti, M., 2007, Acid mine drainage at Mount Morgan, Queensland (Australia): Experimental simulation and geochemical modelling of buffering reactions, in International Mine Water Association, Cagliari, Italy "Water in Mining Environments", 433-436.

Gutiérrez, M., Mickus, K., Camacho, L.G., 2016, Abandoned $\mathrm{Pb}-\mathrm{Zn}$ mining wastes and their mobility as proxy to toxicity: A review: Science of the Total Environment, 565, 392-400.

Huisamen, A., Wolkersdorfer, C, 2016, Modelling the hydrogeochemical evolution of mine water in a decommissioned opencast coal mine: International Journal of Coal Geology, 164, 3-12.

Jamieson, H.E., Walker, S.R., Parsons, M.B., 2015, Mineralogical characterization of mine waste: Applied Geochemistry, 57, 85-105.

Kinniburgh, D.G., Copper, D.M., 2004, Predominance and Mineral Stability Diagrams Revisited: Environmental Science and Technology, 38, 3641-3648.

Klunk, M.A., Damiani, L.H., Feller, G., Rey, M.F., Conceição, R.V., Abel, M., De Ros, L.F., 2015, Geochemical modeling of diagenetic reactions in Snorre Field reservoir sandstones: a comparative study of computer codes: Brazilian Journal of Geology, 45(1), 29-40.

Labarthe-Hernández, G., Tristán-González, M., 1978, Cartografía Geológica San Luis Potosí, S.L.P.: Universidad Autónoma de San Luis Potosí, Instituto de Geología y Metalurgia,
Folleto Técnico, 59, 40 p.

Lindsay, M.B.J., Moncur, M.C., Bain, J.G., Jambor, J.L., Ptacek, C.J., Blowes, D.W., 2015, Geochemical and mineralogical aspects of sulfide mine tailings: Applied Geochemistry, 57, 157-177.

Lottermoser, B.G., 2011, Recycling, reuse, and rehabilitation of mine wastes: Elements, 7 , 405-410.

Luís, A.T., Durães, N., Almeida, S., Ferreira da Silva, E., 2015, Integrating geochemical (surface waters, stream sediments) and biological (diatoms) approaches to assess AMD environmental impact in a pyritic mining area: Aljustrel: (Alentejo, Portugal): Journal of Environmental Sciences, 42, 215-226.

Madzivire, G., Maleka, P.P., Vadapalli, V.R.K., Gitari, W.M., Lindsay, R., Petrik, L.F., 2014, Fate of the naturally occurring radioactive materials during treatment of acid mine drainage with coal fly ash and aluminium hydroxide: Journal of Environmental Management 133, 12-17.

Martínez, O.A., 2015, Movilidad de metales y metaloides en sitios mineros: predicción de impacto en los recursos hídricos: San Luis Potosí, S.L.P., México, Universidad Autónoma de San Luis Potosí, Tesis de maestría, Facultad de Ciencias Químicas, Ingeniería y Medicina, $198 \mathrm{p}$.

Masindi, V., Gitari, M.W., Tutu, H., DeBeer, M., 2015, Synthesis of cryptocrystalline magnesite-bentonite clay composite and its application for neutralization and attenuation of inorganic contaminants in acidic and metalliferous mine drainage: Journal of Water process Engineering, 206, 16 p.

Meima, J.A., Regenspurg, S., Kassahun, A., Rammlmair, D., 2007, Geochemical modelling of hardpan formation in an iron slag dump: Minerals Engineering, 20, 16-25.

Montes, I., 2016, Comportamiento geoquímicoambiental de elementos potencialmente tóxicos (EPT) impactados por un residuo 
minero-metalúrgico histórico: San Luis Potosí, S.L.P., México, Universidad Autónoma de San Luis Potosí, Tesis doctoral en proceso, Instituto de Metalurgia.

Moreno, R., Téllez, J., Monroy, M.G., 2012, Influencia de los minerales de los jales en la bioaccesibilidad de arsénico, plomo, zinc y cadmio en el distrito minero Zimapán, México: Revista Internacional de Contaminación Ambiental, 28(3), 203-218.

Nordstrom, D.K., 2011, Hydrogeochemical processes governing the origin, transport and fate of major and trace elements from mine wastes and mineralized rock to surface waters: Applied Geochemistry, 26, 1777-1791.

Nordstrom, D.K., 2015, Baseline and premining geochemical characterization of mined sites: Applied Geochemistry, 57, 17-34.

Nordstrom, D.K., Campbell, K.M., 2014, Modeling Low-Temperature Geochemical Processes, en Holland H.D., Turekian K.K. (eds.), Treatise on Geochemistry Second Edition: Oxford, Elsevier, 7, 27-68.

Nordstrom, D.K., Blowes, D.W., Ptacek, C.J., 2015, Hydrogeochemistry and microbiology of mine drainage: An update: Applied Geochemistry, 57, 3-16.

Otero-Fariña, A., Gago, R., Antelo, J., Fiol, S., Arce, F., 2015, Surface Complexation Modelling of Arsenic and Copper Immobilization by Iron Oxide Precipitates Derived from Acid Mine Drainage: Boletín de la Sociedad Geológica Mexicana, 67(3), 493-508.

Quispe, D., Pérez-López, R., Acero, P., Ayora, C., Nieto, J.M., 2013, The role of mineralogy on element mobility in two sulfide mine tailings from the Iberian Pyrite Belt (SW Spain): Chemical Geology, 345, 119-129.

Razo, I., Muñoz, R., Cepeda, C., Monroy, M., 2007, Caracterización ambiental de residuos mineros históricos del Distrito Minero de Cerro de San Pedro, San Luis Potosí, México, en Salinas-Prieto, J.C. (ed.), Memoria de la XXVII Convención Internacional de Minería de la Asociación de Ingenieros de
Minas, Metalurgistas y Geólogos de México: Veracruz, 352-357.

Rodríguez-León, M., 2014, Diseño y aplicación de una guía para la caracterización de la hidrología superficial en áreas mineras: San Luis Potosí, S.L.P., México, Universidad Autónoma de San Luis Potosí, Tesis de licenciatura, Facultad de Ingeniería, 157 p.

Romero, F.M., Armienta, M.A., GonzálezHernández, G., 2007, Solid-phase control on the mobility of potentially toxic elements in an abandoned lead/zinc mine tailings impoundment, Taxco, Mexico: Applied Geochemistry, 22, 109-127.

Root, R.A., Hayes, S.M., Hammond, C.M., Maier, R.M., Chorover, J., 2015, Toxic Metal(loid) speciation during weathering of iron sulfide mine tailings under semi-arid climate: Applied Geochemistry, 62, 131-149.

Salas, E.F., 2014, Geoquímica y mineralogía de jales en mina Aurora, Xichú, Guanajuato: México D.F., Universidad Nacional Autónoma de México, Tesis de maestría, Centro de Geociencias, 107 p.

Sánchez-España J., Diez-Ercilla M., 2008, Geochemical modeling of concentrated mine waters: A comparison of the Pitzer ion interaction theory with the ion-association model for the study of melanterite solubility in San Telmo mine (Huelva, Spain), en Stefansson, O. (ed.), Geochemistry Research Advances: Madrid, Nova Science Publishers, $1-25$.

Santos-Jallath J.E., Coria-Camarillo J., HuezoCasillas J.J., Rodríguez-Cruz G., 2013, Influencia de jales mineros sobre el río Maconí, Querétaro, y evaluación del proceso de atenuación natural por dispersión: Boletín de la Sociedad Geológica Mexicana, 65(3), 645-660.

Schaider L.A., Senn D.B., Estes E.R., Brabander D.J., Shine J.P., 2014, Sources and fates of heavy metals in a mining-impacted stream: Temporal variability and the role of iron oxides: Science of the Total Environment, 
490, 456-466.

Secretaria de Economía (SECOFI), 2006,

Muestreo de suelo para la identificación y

la Cuantificación de metales y metaloides, y manejo de la muestra (NMX-AA-132SCFI-2006): México, D.F., Diario Oficial d la Federación, 5 de septiembre de 2006, 33 p. Secretaría de Medio Ambiente y Recursos Naturales (SEMARNAT), 2004, Norma Oficial Mexicana que establece el procedimiento para caracterizar los jales, así como las especificaciones y criterios para la caracterización y preparación del sitio, proyecto, construcción, operación y postoperación de presas de jales (NOM-141SEMARNAT-2003): México, D.F., Diario Oficial de la Federación, 13 de septiembre de 2004, 59 p.

Secretaria deMedioAmbientey Recursos Naturales (SEMARNAT), 2006, Norma Oficial Mexicana que establece las características, el procedimiento de identificación, clasificación y los listados de los residuos peligrosos
(NOM-052-SEMARNAT-2005): México, D.F. Diario Oficial de la Federación, 23 de Junio de 2006, 27 p.

Servicio Geológico Mexicano (SGM), 2008, Monografía Geológico-Minera del estado de San Luis Potosí.

Simate, G.S., Ndlovu, S., 2014, Acid mine drainage: Challenges and opportunities: Journal of Environmental Chemical Engineering, 2, 1785-1803.

Šráček, O., Černík, M., Vencelides, Z., 2013, Applications of Geochemical and Reactive Transport Modeling in Hydrogeology, Palacký University, Olomouc, Faculty of Science, 35-84.

Vázquez, S.E., 2012, Caracterización de un depósito no controlado de residuos mineros y evaluación de su impacto en suelo superficial: San Luis Potosí, S.L.P., México, Universidad Autónoma de San Luis Potosí, Tesis de maestría del programa en geología aplicada, Facultad de Ingeniería, 118 p. 